\title{
Philosophiques
}

\section{L’idéologie dans l’éducation}

\section{Léon Charette}

Volume 3, numéro 2, octobre 1976

URI : https://id.erudit.org/iderudit/203059ar

DOI : https://doi.org/10.7202/203059ar

Aller au sommaire du numéro

Éditeur(s)

Société de philosophie du Québec

ISSN

0316-2923 (imprimé)

1492-1391 (numérique)

Découvrir la revue

Citer ce document

Charette, L. (1976). L’idéologie dans l'éducation. Philosophiques, 3(2), 289-297.

https://doi.org/10.7202/203059ar

Ce document est protégé par la loi sur le droit d'auteur. L'utilisation des services d'Érudit (y compris la reproduction) est assujettie à sa politique d'utilisation que vous pouvez consulter en ligne.

https://apropos.erudit.org/fr/usagers/politique-dutilisation/
Cet article est diffusé et préservé par Érudit.

Érudit est un consortium interuniversitaire sans but lucratif composé de l’Université de Montréal, l’Université Laval et l’Université du Québec à Montréal. Il a pour mission la promotion et la valorisation de la recherche. https://www.erudit.org/fr/ 


\section{Interventions}

\section{L'IDÉOLOGIE DANS L'EDUCATION par Léon Charette}

\section{I - L'INTENTION DE CE PROPOS}

Le but de cet article ne saurait être de faire le point sur la notion d'idéologie, ou de déterminer une fois pour toutes ses rapports avec l'éducation. Les deux notions impliquées sont trop complexes pour justifier un tel optimisme. Il suffirait à l'auteur de contribuer à une certaine clarification préliminaire des termes, et d'essayer d'entrevoir l'un ou l'autre de leurs rapports essentiels, en particulier en ce qui concerne la philosophie pratique.

Ce que le présent texte voudrait surtout suggérer, c'est, d'une part qu'un certain type ou niveau d'idéologie est tout à fait nécessaire et légitime étant donné la fonction éducative de la cité, et, d'autre part, qu'un autre type ou niveau d'idéologie est à proscrire ou à restreindre de plus en plus, à mesure qu'on progresse vers les niveaux universitaires de l'éducation. Car, à ce niveau, l'activité intellectuelle doit se caractériser par la démarche scientifique et non par l'opinion, par la valeur scientifique et non par la valeur de persuasion.

\section{II - LE SENS DES TROIS MOTS}

Précisons dès maintenant que l'idéologie dont nous voulons ici parler est une forme de conscience plus réfléchie que spontanée, renfermant déjà une certaine élaboration doctrinale. Une philosophie et une philosophie de l'éducation sont des élaborations doctrinales. En ce sens, ce sont des idéologies, non spontanées, mais réfléchies. L'idéologie pourrait ainsi être définie comme "un ensemble de représentations collectives par lequel s'affirme une hiérarchie de valeurs ". ${ }^{1}$ Mais on doit encore considérer plus concrètement les différents sens possibles du mot «idéologie ". Et dès

1. J.W. Lapierre, Qu'est-ce qu'une idéologie, dans Les idéologies dans le monde actuel, Paris, Desclée de Brouwer, 1971, p. 14. 
lors, on se rend rapidement compte que, selon les divers critères utilisés pour la classifier, une idéologie peut être limitée à un petit groupe, ou étendue à l'ensemble d'une société ; elle peut être identifiée à une partie seulement, ou à la totalité de la culture ${ }^{2}$ d'un $^{\prime}$ groupe ; elle peut être décrite comme un processus d'aliénation ; elle peut être distinguée de la science ; elle peut être considérée comme un moyen d'action vis-à-vis du pouvoir politique; elle peut être vue enfin (pour arrêter ici l'énumération) comme une interprétation systématique et totalisante de la réalité. Il n'échappera à personne que ces différents sens du mot idéologie ne sont pas toujours exclusifs l'un de l'autre, et qu'ils peuvent se recouper à des degrés divers. Nonobstant la difficulté inhérente au projet, nous nous efforcerons de parler surtout ici de l'idéologie comme élément ou partie de la culture d'une société, car c'est là que peut apparaître le plus clairement son sens légitime.

Le mot " éducation " n'est guère plus simple et pose des problèmes analogues. En effet, ansi que le souligne J. Maritain, le mot éducation possède au moins trois sens distincts. Le premier se rapporte à l'auto-actualisation de la personne. Le second se rapporte " à l'œuvre de formation que les adultes entreprennent auprès de la jeunesse $"^{3}$, (ceci inclut l'influence de la société), et le troisième se rapporte spécifiquement à l'ensemble du système d'enseignement. Au cours de cet article, nous nous arrêterons surtout au second et au troisième de ces sens, selon le contexte de l'exposé.

En ce qui concerne le mot philosophie enfin, nous nous satisferons pour le moment de la description plutôt empirique qu'en donne $\mathrm{S}$. Breton dans un volume connu $\mathrm{u}^{4}$. La philosophie cherche à se caractériser par trois qualités ou attitudes qui sont les suivantes : un effort pour atteindre ce qui est essentiel et fondamental dans les choses ; l'organisation du discours dans un ensemble suffisamment complet; la recherche de la clarté, de la rigueur et de la cohérence dans la présentation: en bref, l'effort de dire ce qui pourrait avoir le sens le plus universel, pour atteindre les choses dans leur essence fondamentale et dans leur totalité, voilà le sens

2. A. et R. Muchielli, Lexique des sciences sociales, Paris, Éd. Soc. Fr., 1969, p. 51. La culture est l'ensemble des institutions et valeurs qui caractérisent une société humaine spécifique.

3. J. Maritain, Pour une pbilosopbie de l'éducation, Paris, Fayard, 1959, p. 18.

4. S. Breton, Situation de la philosophie contemporaine, Paris, E. Vitte, 1959, pp. 13. 16. 
que l'on pourrait donner à cette traditionnelle recherche de la sagesse et aux œuvres dans lesquelles cette recherche prend forme.

\section{III — LE PROBLÈME IMMÉDIAT}

Ces trois termes étant présentés, le problème auquel je voudrais madresser est le suivant: un vocabulaire initialement marxiste, mais largement répandu dans les sciences humaines, conduit de plus en plus les adeptes de ces sciences et de la philosophie, à croire que leur entreprise est irrémédiablement entachée d'idéologie. Ce fait constituerait un vice tellement profond et indéracibale qu'il s'en faudrait de peu, selon plusieurs, pour qu'on tombe dans l'historicisme et le relativisme le plus pur. D'autres penseurs, marxistes surtout, croient pouvoir éviter la difficulté en recourant à la distinction qui oppose la science à l'idéologies. Qu'en est-il donc? Les sciences humaines et la philosophie sontelles irrémédiablement condamnées à formuler et communiquer seulement des visions déformantes et interchangeables de la réalité? Celui qui enseigne ces disciplines, et l'éducateur en général, sont-ils autre chose que de simples instruments inconscients de propagande ou d'endoctrinement?

\section{IV - L'IDÉOLOGIE DANS LA SCIENCE, L'ÉDUCATION, LA POLITIQUE}

Il conviendrait de nous dissocier, au moins au point de vue méthodologique, de la distinction science-idéologie au sens marxiste. Premièrement parce que le fondement de cette distinction n'est pas évident ; deuxièmement parce que la science au sens marxiste effectue une réduction de la notion même de science ${ }^{\sigma}$; et troisièmement parce que cette science elle-même n'est pas exempte de présupposés idéologiques se rapportant à une certaine vision du monde. Par ailleurs, si nous nous reportons aux discussions actuelles sur la notion même de science, les conclusions de K. Popper et, plus récemment, de T.S. Kuhn et I. Lakatos', semblent également incapables de nous fournir un critère efficace pour distinguer la science de l'idéologie. En effet, il n'est guère éclairant pour

5. H. Lefebvre, Problèmes actuels du Marxisme, Paris, P.U.F., 1958, p. 44.

6. E. Simard, Communisme et science, Québec, P.U.L., 1963, p. 283 (texte de J. Staline).

7. R.F. Baum, "Popper, Kuhn, Lakatos : A Crisis of Modern Intellect ", dans The Intercollegiate Review, Spring 1974, Vol. 9, No. 2, p. 106. 
notre propos de constater que même dans les sciences de la nature, il peut se produire une véritable compétition entre des paradigmes ou modèles scientifiques incorporant différents visions du monde, et que le choix de théories fondamentales par le savant tient surtout à des raisons de sociologie et de psychologie individuelle. Le roc ferme des sciences de la nature semble donc en voie de s'effriter et de nous priver du critère qui aurait pu servir de ligne de démarcation entre les sciences et l'idéologie. Si, enfin, nous laissons de côté le modèle scientifique des systèmes formels, c'est, d'une part, que les limites de la formalisation, depuis Gödel, sont assez connues $^{8}$, et, d'autre part, que l'objet de notre étude ne saurait se prêter, du moins pour le moment, à une formalisation satisfaisante. Il vaut certainement mieux, comme le suggère $M$. Lagueux ${ }^{9}$, ne pas trop insister sur l'opposition science-idéologie.

Reprenons donc la question d'une autre façon, cette fois en partant de la notion d'idéologie. On n'aura sans doute aucune difficulté à faire admettre que l'idéologie entretient toujours quelque rapport avec les contenus de l'enseignement, avec la transmission des valeurs qui accompagne l'enseignement de toute science, avec les options qui conditionnent les types et les niveaux d'enseignement, avec les visées de toute formation sociale et civique, avec l'effort de persuasion mis en cuvre par toute éducation au sens large, avec cette culture enfin, dont l'éducation et l'idéologie ellemême font partie. Le Rapport Faure ${ }^{10}$, par sa recommandation de faire de la cité tout entière une Cité éducative, reconnaît bien l'action consciente et délibérée qu'un État doit jouer auprès de ses citoyens et que toute institution sociale joue, même inconsciemment ou indirectement. On ne connaît personne qui s'offusquerait d'une telle nécessité, ou qui en contesterait la légitimité.

En effet, les chercheurs reconnaissent de plus en plus qu'une société ne saurait se passer d'idéologie ${ }^{11}$, parce qu'elle doit se représenter à elle-même, c'est-à-dire se formuler, puis se communi-

8. J. Ladrière, Les limites de la formalisation, dans Logique et Connaissance scientifique, Paris, Gallimard, Encyclopédie de la Pléiade, 1967, pp. 312-332.

9. M. Lagueux, L'usage abusif du rapport science / idéologie, dans Culture et langage, Montréal, Hurtubise HMH, 1973, p. 226.

10. Rapport Faure, Apprendre à Etre, Paris, Unesco, Fayard, 1972, p. 186.

11. J. Ellul, Propagandes, Paris, Armand Colin, 1962, ch. 5, début. M. Amyot et al., Les idéologies dans le monde actuel, Paris, Desclée de Brouwer, 1971, p. 173, (Article de J. Lacroix). 
quer ; elle doit être organisée et doit agir dans un contexte et des circonstances particulières et précises. La politique est du domaine de l'action, et puisque toute tendance vers l'action vise à rejoindre le concret et ses conditions particulières, la politique ne peut dépendre exclusivement de vues théoriques sur les hommes et la société. Elle inclut inévitablement aussi des vues pratiques plus ou moins particulières, qui sont le lieu naturel de l'idéologie. Comme tout ensemble de concepts ordonnés à l'action concrète et impliquant le désir ${ }^{12}$ de biens particuliers et contingents, la politique et l'idéologie simplifient; elles tendent au particulier et à l'opinion. C'est pourquoi elles comportent inévitablement une part de persuasion, de rhétorique ou de propagande. Lors d'une conférence récente, le philosophe $P$. Ricœur attribuait justement à l'idéologie un premier rôle, tout à fait positif, qui est sa fonction intégrative et médiatrice au sein de la société. En se sens, concevoir la politique sans idéologie ou sans rhétorique apparaît impossible. Nous parlons évidemment ici de la politique en elle-même, et non de la façon dont elle peut être pratiquée dans des circonstances particulières bien définies. À ce même point de vue de la chose en elle-même, ce qui est dit ici de la politique pourrait être dit tout autant de l'éducation, car elle ne se conçoit et n'existe pas non plus, sans idéologie.

\section{V - LES NIVEAUX OU TYPES DE DISCOURS DE L'IDÉOLOGIE}

Cependant, dire d'une réalité qu'elle est idéologique ou qu'elle accepte de se dire idéologique ne signifie pas qu'on prononce sur elle un jugement sans appel. Les viccissitudes de la science, auxquelles nous avons fait allusion plus haut, nous invitent à plus de modération. De plus, il y a idéologie et idéologie. En effet, comme toute rationalisation se rapportant à l'action, l'idéologie peut se présenter à différents degrés de proximité de l'action concrète. Et, de ce fait, il y a plusieurs niveaux d'idéologie, ou de langage idéologique, dont le statut épistémologique est peut-être différent. Considérons, par exemple, la série ${ }^{13} \mathrm{~d}^{\prime}$ affirmations suivantes :

1. L'homme est naturellement sociable.

12. F. Dumont, Les idéologies, P.U.F., Collection SUP, 1974, p. 43.

13. Pour d'autres exemples, voir L.P. Brown, Ideology, Baltimore, Penguin Books Ltd., 1973, pp. 40-69. 
2. L'autorité est nécessaire à la société.

3. L'autorité doit veiller au bien commun.

4. L'autorité politique peut revêtir plusieurs formes.

5. La forme d'autorité démocratique est la meilleure.

6. Tel parti politique peut former un meilleur gouvernement.

7. Tel programme politique est excellent.

8. Telle décision politique semble souhaitable.

9. Les modalités de cette décision paraissent bonnes.

L'attitude politique concrète du citoyen ou d'un groupe de ci. toyens peut incoroprer toutes ou seulement quelques-unes de ces affirmations, ou encore aucune (comme, peut-être, les Témoins de Jéhovah) ${ }^{14}$. Quoiqu'il en soit, le statut épistémologique de ces propositions n'est pas identique, pas plus que l'utilisation idéologique que peuvent en faire les individus ou les groupes. Par conséquent, il apparaît abusif dans certains contextes de parler d'idéologie en général, sans identifier le type ou niveau d'idéologie au sujet duquel on veut spécifiquement discourir. Il apparaît également abusif de toujours parler d'idéologie au seul sens d'une vision essentiellement déformante et illusoire. $\AA$ ce compte, toute connaissance, lorsqu'elle s'exprime dans l'ordre pratique, serait essentiellement idéologique, et donc mensongère. Or, du fait qu'un discours quelconque soit toujours exprimé par une subjectivité, il ne s'ensuit pas que ce discours soit intégralement subjectif, c'est-à-dire qu'il n'ait qu'une valeur ou une portée subjective. En outre, on ne voit pas vraiment ni pourquoi, ni comment, la seule façon de s'arracher à l'idéologie serait de construire une réflexion sociale radicalement critique et totale. En effet, cette position nie d'abord la distinction entre le spéculatif et le pratique. Ensuite, elle fait dépendre la valeur de la connaissance spéculative d'une quelconque justification de la connaissance pratique. En dernier lieu, elle considère comme identiques les conditions de validité ou de vérité de la connaissance spéculative de la connaissance pratique ${ }^{i s}$. Le rapport entre l'idéologie et la réalité apparaît plus complexe. Il faut plutôt penser qu'il

14. John A. Hardon, The Protestant Churcbes of America, New York, a Doubleday Image Book, 1969, p. 333.

15. F. Dumont, Les idéologies, P.U.F., Collection SUP, 1974, p. 46. 
ne se réduit pas au rapport du faux au vrai ; qu'il est plus ambigü : il est de "reconnaissance et de méconnaissance" "d'illusion et d'allusion" (Althusser) $)^{16}$.

À cet égard, la position de L. Althusser nous paraît relativement juste, puisqu' elle permet d'inclure le rôle positif du premier niveau de l'idéologie. Il faut encore ajouter ceci. Dans nos remarques précédentes, il aurait fallu préciser les rapports respectifs de l'art et de la science avec l'idéologie. Car, la pratique (art), en éducation par exemple, diffère nécessairement de la vision ou conception (discipline-science) qui l'inspire. Il en est de même en ce qui concerne la politique, la philosophie et la science. La conception qui les anime, toute idéologique qu'elle soit, sera toujours différente de l'utilisation idéologique qu'on peut en faire. Elle ne s'y réduit pas.

D'un autre point de vue, il faudrait encore revoir la distinction science-idéologie, parce qu'elle n'apparaît pas rigoureusement équivalente à la distinction science-opinion. La raison en est non seulement que la science peut elle aussi comporter des affirmations dont la certitude n'est que probable, mais encore, que l'idéologie elle-même pourrait bien incorporer, à un certain niveau, des affirmations dont la certitude paraît plus que probable. Par exemple, les quatre premières affirmations de la série énoncée dans la section précédente ne sauraient être reléguées purement et simplement dans le domaine de l'opinion ${ }^{17}$. Nous croyons, pour notre part, que le vocabulaire d'une certaine philosophie morale traditionnelle renferme ici une distinction qui est loin d'avoir perdu toute son utilité. En effet, à partir, d'une part, du type de principes incorporés dans le discours, et, d'autre part, de la plus ou moins grande proximité des affirmations par rapport à l'action concrète, la philosophie morale scolastique distinguait entre le discours « spéculativement pratique " et le discours " pratiquement pratique $"{ }^{18}$. Or, comme l'idéologie constitue un discours d'ordre pratique, elle peut se situer à chacun des deux niveaux mentionnés ci-dessus. Au premier niveau, le discours idéologique jouit de la certitude qui ca.

16. M. Rafie, Idéologie et sciences bumaines, dans Culture et langage, Montréal, Hurtubise HMH, 1973, p. 234.

17. Sur le "noyau " de savoir inclus dans toute idéologie, voir F. Dumont, Les idéologies, PUF., Collection SUP, 1974, p. 110 ; L.B. Brown, Ideology, Baltimore, Penguin Books Ltd., 1973, p. 176.

18. J. Maritain, Les degrés du savoir, Paris, Desclée de Brouwer, 1946, p. 885. 
ractérise les affirmations de la science morale (au sens aristotélicien de l'expression), ni plus, ni moins. Au second niveau, le discours idéologique, de par son caractère complexe et rapproché de l'action, ne semble apte à produire rien de plus que l'opinion, quelles que soient par ailleurs la nécessité et la légitimité de ce discours. L'ensemble qui en résulte, c'est-à-dire l'idéologie comme tout, est lui aussi du niveau de l'opinion.

\section{VI - QUELQUES APPLICATIONS}

Si l'on essayait maintenant d'appliquer quelques-unes des distinctions qui précèdent soit à l'éducation, soit à la philosophie, il faudrait commencer par dire que toutes deux, en tant qu'on en fait usage ou qu'on les exerce, incorporent les deux niveaux de l'idéologie et se trouvent, à ce titre, particulièrement vulnérables. Toutes deux le paraissent même d'autant plus qu'elles peuvent se joindre à des idéologies tout à fait différentes, au plan du discours pratiquement pratique. Mais alors, le discours complexe qui en résulte n'a plus que valeur d'opinion. Ce discours rassemble des éléments hétérogènes; il explique et simplifie ; il justifie et rationalise ; il déforme sans cesser d'être nécessaire et légitime. C'est à ce niveau que peut apparaître surtout l'idéologie qui est mensonge et illusion.

Par ailleurs, si l'on essaie d'envisager l'éducation et la philosophie en elles-mêmes, et comme s'exprimant au niveau spéculativement pratique, le discours qui en résulte peut encore être qualifié d'idéologique, mais ce ne peut plus être au sens qui précède. D'une part, l'éducation et la philosophie apparaissent ici nettement irréductibles à l'idéologie, du fait même de cette compatibilité avec des idéologies différents au plan du discours pratiquement pratique. D'autre part, comment soutenir qu'une conception de l'éducation ou

qu'une philosophie ne vaut que comme production culturelle et se révèle inexplicable de part en part par les relations qu'elle entretient avec l'environnement social, politique, historique :!"

À ce niveau, de l'éducation et la philosophie s'efforcent surtout d'élaborer un discours qui esquisse un idéal à poursuivre. Et à ce titre, elles se rapprochent beaucoup plus de l'utopie que de

19. E. Borne, Idéologie et philosophie, dans Les idéologies dans le monde actuel, Paris, Desclée de Brouwer, 1971, p. 81. 
l'idéologie ${ }^{20}$. Elles apparaissent davantage comme un "contreidéologie " visant inlassablement d̀ produire la "conscience claire ${ }^{21}$, non seulement pour une classe sociale donnée, mais à l'intérieur de la société toute entière. Voilà un rôle que la philosophie n’a jamais dédaigné.

Les remarques qui précèdent ne prétendent aucunement à l'exhaustivité, la profondeur ou l'érudition. Elles expriment simplement une certaine perplexité, un certain étonnement, devant ce qu'il est convenu d'appeler la crise de la raison contemporaine. Elles ne cherchent qu'à promouvoir un dialogue constructif, à partir de notions peut-être anciennes, mais dont l'utilité est apte à être renouvelée.

Université d'Ottawa

20. K. Mannheim, Ideology and Utopia, (trad. de l'allemand), New York, Harvest Book, 1936, p. 192.

21. G. Rocher, Sociologie générale, Montréal, HMH, tome 3, 2e éd., 1969, p. 380. 